\title{
Motion and Disparity Estimation with Self Adapted Evolutionary Strategy in 3D Video Coding
}

\author{
S. Adedoyin, W.A.C. Fernando, A. Aggoun, K.M. Kondoz
}

\begin{abstract}
Real world information, obtained by humans is three dimensional (3-D). In experimental user-trials, subjective assessments have clearly demonstrated the increased impact of 3-D pictures compared to conventional flat-picture techniques. It is reasonable, therefore, that we humans want an imaging system that produces pictures that are as natural and real as things we see and experience every day. Three-dimensional imaging and hence, 3-D television (3DTV) are very promising approaches expected to satisfy these desires. Integral imaging, which can capture true $3 D$ color images with only one camera, has been seen as the right technology to offer stress-free viewing to audiences of more than one person.

In this paper, we propose a novel approach to use Evolutionary Strategy (ES) for joint motion and disparity estimation to compress $3 D$ integral video sequences. We propose to decompose the integral video sequence down to viewpoint video sequences and jointly exploit motion and disparity redundancies to maximize the compression using a self adapted ES. A half pixel refinement algorithm is then applied by interpolating macro blocks in the previous frame to further improve the video quality. Experimental results demonstrate that the proposed adaptable ES with Half Pixel Joint Motion and Disparity Estimation can up to $1.5 \mathrm{~dB}$ objective quality gain without any additional computational cost over our previous algorithm. Furthermore, the proposed technique get similar objective quality compared to the full search algorithm by reducing the computational cost up to $90 \%$.
\end{abstract}

Keywords: 3D Video, Motion Estimation, Video coding

\section{INTRODUCTION}

$\mathrm{T}$ here is growing evidence that three-dimensional (3D) imaging techniques will have the potential to establish a future mass-market in the fields of (television, video game) and communications (desktop video conferencing). One much discussed application is $3 \mathrm{D}$

Steven Adedoyin is with the University of Surrey, Guildford, GU2 7XH, UK (e-mail: S. Adedoyin@surrey.ac.uk).

W.A.C.Fernando is with the University of Surrey, Guildford, GU2 7XH, UK (e-mail: W.Fernando@surrey.ac.uk).

A.Aggoun is with the school of engineering and design of Brunel University UB8 3PH, UK (amar.aggoun@brunel.ac.uk)

A.M. Kondoz is with the University of Surrey, Guildford, GU2 7XH,UK (e-mail: A.Kondoz@surrey.ac.uk).

Contributed Paper

Manuscript received October 15, 2007 television. Many different approaches have been adopted in attempts to realize free viewing (auto-stereoscopic) 3D TV. True auto-stereoscopic 3D visualization systems exhibiting parallax in all directions, which allow accommodation and convergence to work in unison, are ideally required. Holography, which demonstrates these characteristics, continues to be researched by different groups in an effort to produce full color realistic spatial images. However, the requirements for coherent light sources, dark room conditions and high mechanical stability during recording reduce the practical utility of holographic technique for general 3D spatial video imaging applications.

Following the introduction of digital broadcast, 3D TV is seen as the next major step forward [2][3]. Most of the research for the next generation of audio-visual systems has centred around stereoscopic 3D imaging, which requires multiple camera capture and multiple view displays [4]. In most of these designs, a lenticular sheet decoder or scanning aperture is exploited to enable transmission of image data into the left and right eyes by spatial or temporal multiplexing. However, such displays are not truly spatial since they exclude vertical parallax and rely upon the brain to fuse the two disparate 2D images to create the 3D sensation. A fundamental limitation of stereo systems is that they tend to cause eye strain, fatigue and headaches after prolonged viewing as the user is required to focus to the screen plane but converge their eyes to a point in space, producing a very unnatural situation [5].

Integral imaging is a technique that is capable of creating and encoding a true volume spatial optical model of the object scene in the form of a planar intensity distribution by using unique optical components[6][7][8][9][10][11][12]

[13][14]. It is akin to holography in that $3 \mathrm{D}$ information recorded on a 2-D medium can be replayed as a full 3D optical model, however, in contrast to holography, coherent light sources are not required. This conveniently allows more conventional live capture and display procedures to be adopted. With recent progress in the theory and microlens manufacturing, integral imaging is becoming a practical and prospective 3D display technology and is attracting much interest in the $3 \mathrm{D}$ area [6][7][8][9][10][11][12][13][14].

TV based on 3D integral imaging video technology, that requires only one camera, will be attractive to service 
providers because it will seamlessly provide the added value of three-dimensional realism. This approach is attractive to service providers because it avoids the cumbersome setting up of more than one camera that other 3D capture techniques employ. 3D integral imaging encoded video can be designed to be scalable with $2 \mathrm{D}$ video and can be encoded efficiently so as to economically provide attractive high value services over high value systems. Thus, the development of a 3D integral imaging TV system will also demonstrate how added-value broadband services of this type can be delivered, providing benefit to designers of these type of services in the future. However, lots of work needs to be done in this area specially in the compression of $3 \mathrm{D}$ integral imaging video.

In $3 \mathrm{D}$ integral video compression motion estimations is the most critical part as it takes up to $70 \%-90 \%$ of the encoding time and greatly reduces the amount of data needed to represent a video sequence. An exhaustive block matching algorithm is the conventional method used to conduct motion estimation. This searches possible position within a specified area. The Full Search block motion estimation (BMA) will find the best match by using a matching criterion such as SAD to compare every block to the current block. However such a method entails a high computational cost since it involves several view points.

In this paper, we propose a joint motion and disparity motion estimation with a half pixel refinement technique using an adaptable evolutionary strategy for 3D integral imaging video. Rest of the paper is organised as follows. In section 2, we summarize some related work on 3D integral imaging. The proposed algorithm is presented in section 3. Section 4 presents some simulation results and a detailed analysis. Finally, the conclusions are given in section 5 .

\section{RELATED WORK}

In 1908 Lippmann introduced a system known as integral photography[15]. This is a technique for creating full color optical models, which exist in space independently of the observer. They are autostereoscopic and the images exhibit continuous parallax throughout the viewing zone. The continuous nature of the images produced with this method eliminates the effect of 'cardboarding' (flattening of objects into discrete depth planes) and flipping (a visible effect created by moving between image fields) present in multi-view stereoscopic systems.

The development of integral 3D imaging has been inhibited by: (i) the inherent difficulty in manufacturing high quality microlens arrays; (ii) orthoscopic integral 3D imaging is a two stage process; (iii) images rely upon the resolving capability of the microlens array; (iv) Okoshi [2] suggested a bandwidth of $42 \mathrm{GHz}$ is necessary for transmission; (v) published theory indicates that integral
3D images will not reconstruct at the resolutions currently attainable in modern electronic display devices[3].

A three dimensional imaging arrangement based around Lippmann's integral photography technique has been reported [6][7][8][9] and is shown in figure 1. A single 'camera' unit encodes a true optical model of the scene as a single flat intensity distribution suitable for electronic capture. A flat panel display for example one using LCD technology is used to reproduce the captured intensity modulated image and a microlens array re-integrates the captured rays to replay the original scene in full color and with continuous parallax in all directions. This system offers the potential for stress-free viewing by more than one person.

It is possible to capture integral 3D images electronically using a commercially available CCD array. This form of capture requires a high resolution CCD together with specialised optical components to record the micro-images fields produced by precision micro-optics.

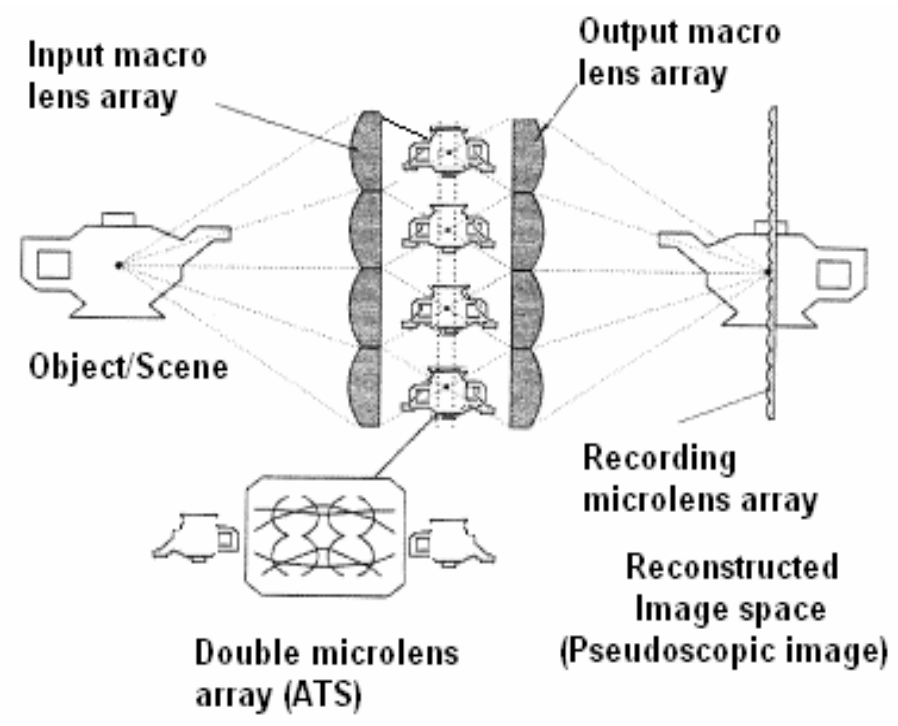

Figure 1 An advanced Integral Imaging system

The system would record live images in a regular block pixel pattern. The planar intensity distribution representing an integral image is comprised of 2D array of MxM micro images due to the structure of the microlens array used in the capture and replay. The resulting 3D images are termed Omnidirection Integral Images (OII) and have parallax in all directions. The rectangular aperture at the front of the camera and the regular structure of the hexagonal microlenses array used in the hexagonal grid (recording microlens array) gives rise to a regular 'brick structure' in the intensity distribution.

In this paper, the simulation work is carried out on unidirectional integral images (UII) which are obtained by using a special case of the integral 3D imaging system where 1D cylindrical microlens array is used for capture 
and replay instead of a 2D array of microlenses. The resulting images contain parallax in the horizontal direction only. Figure 2(a) shows an electronically captured unidirectional integral 3D image and figure 2(b) shows a magnified section of the image. The $\mathrm{M}$ vertically running bands present in the planar intensity distribution captured by the integral $3 \mathrm{D}$ camera are due to the regular structure of the $1 \mathrm{D}$ cylindrical microlens array used in the capture process.

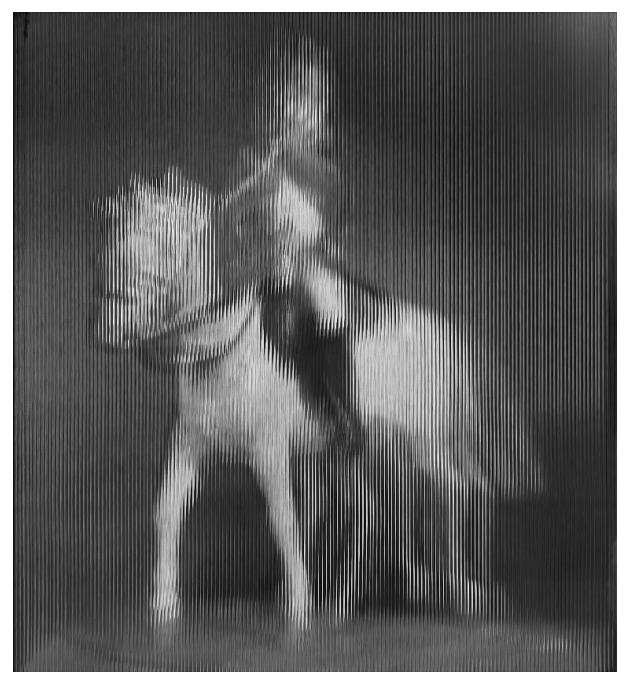

(a)

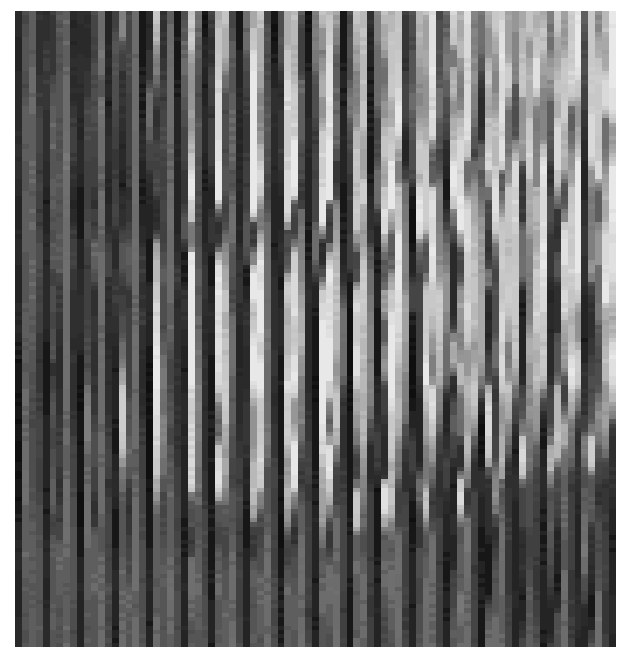

(b)

Figure 2 An electronically captured unidirectional integral image a) Full. b) Magnification

Due to the large amount of data required to represent the captured 3D integral image with adequate resolution, it is necessary to develop compression algorithms tailor to take advantage of the characteristics of the recorded integral image. The planar intensity distribution representing an Omnidirectional Integral Image is comprised of 2D array of sub-images due to the structure of the microlens array used in the capture and replay. The structure of the recorded integral image intensity distribution is such that a high cross correlation in a third domain, i.e. between the micro-images produced by the recording microlens array, is present. This is due to the small angular disparity between adjacent microlenses. In order to maximise the efficiency of a compression scheme for use with the integral image intensity distribution, both inter and intra sub-image correlation should be evaluated.

In the last decade, a Three Dimensional Discrete Cosine Transform (3D-DCT) coding algorithm has been developed for compression of still 3D integral images [19][20][21][22]. The algorithm took advantage of the redundancies within the microlens sub-images (intra subimage coding) and the redundancies between adjacent microlens sub-images (inter sub-image coding). The use of intra and inter sub-image coding resulted in much better results than those obtained with the baseline JPEG with respect to both image quality and compression ratio. The main advantage of using transform coding is that integral 3D images are inherently divided into small nonoverlapping blocks referred to as microlens sub-images. This leads to high compression with less blocking artefacts.

\section{Proposed 3D Video Encoder}

In $3 \mathrm{D}$ integral video, each viewpoint video sequence represents a unique recording direction of the object scene. Therefore, the 3D integral video sequence can be separated into its respective distinctive viewpoint videos. Figure 3 illustrates the viewpoint extraction for a lenticular video of 4 distinctive viewpoints. Columns of pixels formed by each micro lens representing the similar view points are placed near to each other to form the viewpoint images. These viewpoint video sequences have lots of correlations and can be exploited from the motion and disparity estimation.

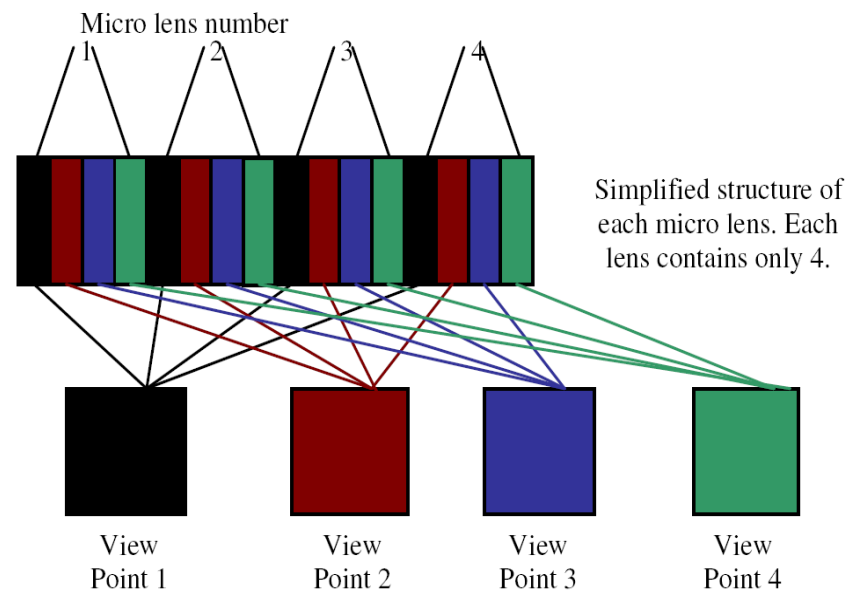

Figure 3 Viewpoint extraction 
S. Adedoyin et al.: Motion and Disparity Estimation with Self Adapted Evolutionary Strategy in 3D Video Coding

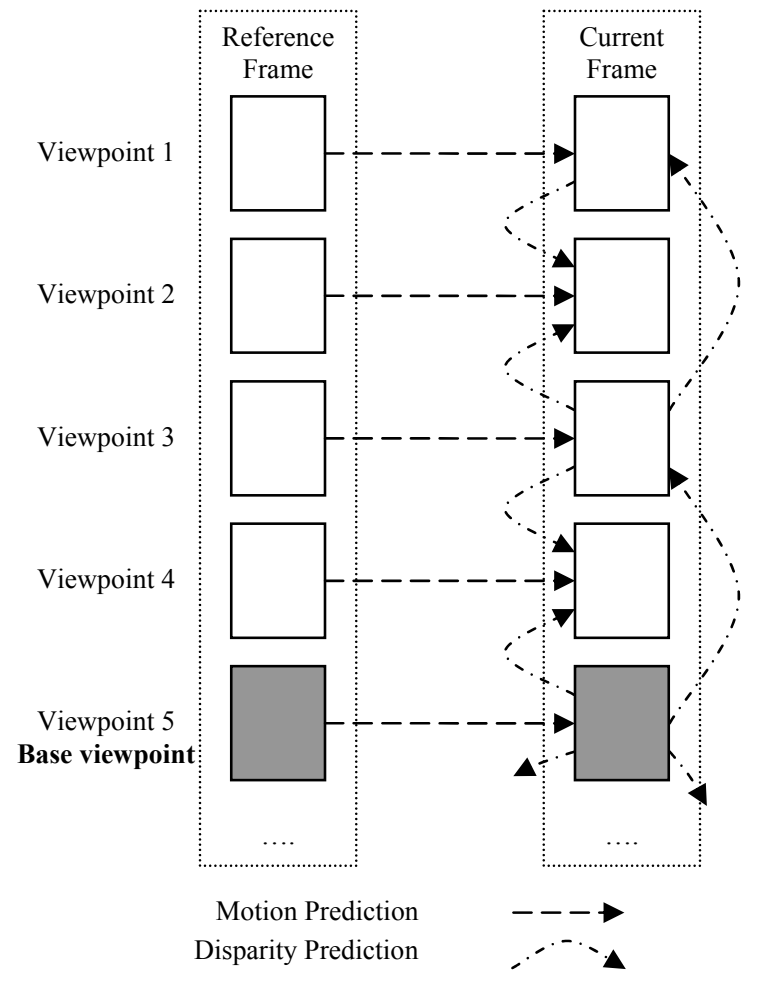

Figure 4 Proposed motion and disparity estimation technique

The best motion vectors for each viewpoint can be found by applying a conventional block-matching algorithm. However such a technique would be too complex and time consuming. Since viewpoint images are captured by slightly different viewing angles, there is a great deal of redundancies. Therefore, it is advantageous to find a proper set of motion vectors only for a single viewpoint and utilize this correlation to minimize the overall coding complexity. Compression efficiency can be maximized if disparity correlations amongst the viewpoints are also considered. Exploitation of such additional redundancies, however, increases the computational complexity further. Therefore, there is a great deal of necessity for an efficient technique to exploit most of the existing correlations within lenticular video at an acceptable computational cost. Following this argument, we proposed a joint motion and disparity estimation algorithm based on ES to minimize the computational cost[1]. In this paper we extend the previous work by considering self adaptation with half pixel refinement to further improve the performance.

Figure 4 illustrates the basic coding architecture for motion and disparity estimation. The middlemost viewpoint is considered as the base view point and the rest of the view points are motion and disparity compensated jointly considering the motion compensated viewpoint as the base viewpoint. After coding the base viewpoint (i.e. Viewpoint 5) with respect to the base viewpoint of the reference frame, adjacent viewpoints are coded taking the corresponding viewpoint from the reference frame and the reconstructed version of the base viewpoint of the current frame as references. This process is repeated for the other viewpoints in the sequence.

In this paper we use $(\boldsymbol{\mu}+\boldsymbol{\lambda})$-Evolutionary Strategy [26] like in our previous paper [1]. In the proposed ES, each chromosome represents three elements of a motion vector. These are $\mathrm{x}, \mathrm{y}$ which defines the horizontal and vertical components of a motion vector, and the reference which defines if the vector resides in a previous frame (in case of motion compensation) or viewpoint (in case of a disparity compensation). Each of these components has both an object and strategy gene associated to it. The values of $x$ and $y$ are determined from the search window size. For example, if the search window is within the range $[-8,8]$, then the value of object gene can take any integer number inside the range. Strategy gene determines if local or global search will be carried out. Smaller value of strategy gene mean the search becomes more localised.

The importance of a chromosome in a population is defined by the fitness function. This function divides the strongest from the weak. If a chromosome is deemed strongest it will be the $1^{\text {st }}$ selected chromosome for producing offspring within the next generation, as objects with good genes are considered to produce the finest offspring. The Fitness function is calculated based on the Sum of Absolute Difference (SAD). The Sum of Absolute difference is a widely used search criterion for motion estimation and is calculated by using the following formula:

$$
\begin{aligned}
& \operatorname{SAD}(x, y, r, s)= \\
& \sum_{i=1}^{i=8} \sum_{j=1}^{j=8}\left|A_{(x+i, y+j)}-B_{((x+r)+i,(y+s)+j)}\right|
\end{aligned}
$$

Where $x, y$ denote the target block $\mathbf{A}$ and $r, s$ donates the offset from the block in the reference frame $\mathbf{B}$. The vertical and horizontal components of a given block type is represented by $i, j$. A chromosome in ES represents the offset. Each chromosome in newly generated population is evaluated using fitness function. The chromosome with the lowest SAD will be the fittest individual within the population. Evolutionary strategy operators have been defined as in [1].

\section{A. Population Generation}

In order to reduce the number of generations required to obtain a satisfactory solution, the initial population includes a number of pre-defined chromosomes. This includes the $(0,0)$ motion vector, in case of zero biased motion i.e. a static background. As all motion is not zero biased, the population also includes the fittest chromosomes from spatial adjacent blocks, temporal adjacent blocks and includes the disparity related blocks. 
These blocks are selected as the motion vector correlations between these blocks tend to be similar.

The remaining members of the population are made up of randomly generated chromosomes. Without the predefined chromosomes the ES algorithm would still find the correct match, but with an increase in the number of generations hence more search points.

The size of initial population is set to 30 , giving the ES algorithm a good representation of adequate regions within the search window. During the selection process the population size alternates, it combines the parents and the offspring to determine which individuals are deemed unfit. These individuals are then purged from the population, before new offspring's are created.

\section{B. Mutation}

The Mutation rate defines the percentage of genes to be mutated in a newly generated population. These genes are randomly chosen. The higher the mutation rate the higher the number of offspring thus the higher the number of search points. Experimental results show that a mutation rate of $8.5 \%$ is sufficient enough. In general an offspring is denoted by,

$$
x_{\text {offspring }}^{n}=x_{\text {parent }}^{n}+\mathrm{N}\left(0, \sigma_{\text {parent }}^{n}\right)
$$

Where $N\left(0, \sigma_{\text {parent }}^{n}\right)$ an M-dimensional vector of random Gaussian numbers with zero mean and standard deviations $\sigma . \quad x_{\text {offspring }}^{n}$ represents a newly created object gene through mutation and $x_{\text {parent }}^{n}$ represents a randomly chosen gene from the population. In this illustration $\sigma$ represents the strategy gene, which is dependant on the self-adaptation algorithm explained later.

\section{Self Adaptation}

One of the main benefits of ES is its self adaptation techniques. Self adaptations benefits can be seen in other ES research. Experimental results showed that the strategy gene and mutation rates must decrease over time. Given that large parts of the search space needs to be explored to determine areas with low SAD's. As the search advances and the best possible values approach smaller values for the strategy gene are needed to adjust chromosomes, so that the optimal match is found.

The method employed in alternating the strategy gene and ensuring that good regions is based on Rechenberg 1/5[26] success rule and Greenwood and Zhu Modified's Self Adaptation [27]. Rechenberg 1/5 success rule states that the ratio of successful mutations (those in which the child is fitter than the parent) to all mutations should be $1 / 5$. Therefore if the ratio is greater than $1 / 5$ the strategy gene should be increased to find alternate good regions and if it is less the strategy gene is decreased to concentrate the search more around the given object gene. A major stumbling block with the $1 / 5$ success rule is that it causes ES to become stuck at a local minimum. This is because over time the percentage of successful mutations will drop below $1 / 5$. Thus causing $1 / 5$ success rule to reduce the strategy gene, which ensures a reduction in the search space, this in turn causes the search to be stuck on a local minimum and miss the global minimum. In the proposed algorithm we used the following method shown in [27] to overcome this problem by modifying the strategy gene.

$$
\sigma^{\text {new }}= \begin{cases}\min (\sigma / c, D) & \text { if } p_{s}>1 / 5 \\ \sigma & \text { if } p_{s}=1 / 5 \\ \sigma . c & \text { if } 1 / 20 \leq p_{s}<1 / 5 \\ \min (2 \sigma, D) & \text { if } p_{s}<1 / 20\end{cases}
$$

$p_{s}$ donates the number of total successful mutations over a number of generations. $\mathrm{C}$ is a constant its value is 0.6 , and it is used to increase or decrease the strategy gene, $\sigma^{\text {new }}$ represents the modified strategy gene, and $\mathrm{D}$ represents half the diameter size of the search space. In this case the search range is 8 therefore $D$ will also equal 8. D is used as an upper limit so that $\sigma$ never exceeds it. If $p_{s}$ is less than $1 / 20$ then the strategy gene is increased to stop the search from falling into local minima.

\section{Half Pixel Refinement}

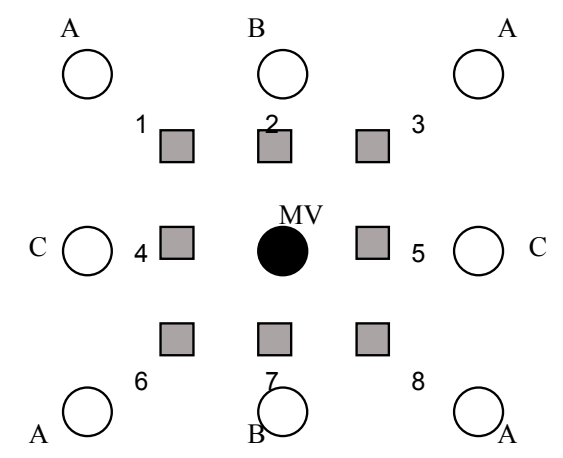

Figure 5 Half pixel search positions around the pixel MV

After a block matching algorithm has found the best matching motion vector, it is likely that a better match can be found by searching the interpolated region surrounding the block in the reference frame. This is known as sub sampling. Half pixel refinement is a form of sub sampling. Testing the 8 surrounding sub pixel positions in the reference frame each with a half pixel distance around the obtained motion vector 
as illustrated in figure 5 . These positions are acquired by using the formula as described below.

$$
\begin{aligned}
& \text { Positions } 2 \& 7=(B+M V) / 2 \\
& \text { Postions } 4+5=(C+M V) / 2 \\
& \text { Positions } 1,3,6 \& 8=(M V+A+B+C) / 4
\end{aligned}
$$

The formula illustrated above is then used to construct an interpolated block. The SAD of this block is then compared to the original. If it is less, it is then chosen as the new motion vector.

\section{RESULTS}

Proposed adaptable ES with joint motion and disparity estimation algorithm is implemented in a 3D-DCT integral image codec based on the architecture described in [19]. An adaptive arithmetic coder is used for the entropy coding and the quantizer step size ranged from $10-50$. A population size of 30 was used for ES. Peak Signal-toNoise Ratio (PSNR) of the luminance signal and its bit rate are used to measure the objective quality. For all simulations, all other simulation parameters have been selected as in [1].

Figures 6-10 show the objective quality comparison of the proposed ES based joint motion and disparity estimation technique with half pixel refinement (denoted as proposed) for the 'Room' integral video test sequence of image size $512 \times 512$ against three reference cases namely:

(i)

Motion only full search (FS-MOTION) motion compensated prediction is used and the motion vectors are calculated using the full search algorithm.

(ii) Motion only ES search (ES-MOTION) - as above except full search is replaced with ES search.

(iii) Joint motion and disparity full search (FSJM\&D) - joint motion and disparity compensated prediction.

(iv) Joint motion and disparity with ES (ESJM\&D).

Figure 6-8 depicts the relative performance of the above algorithms for selected viewpoints and Figure 9-10 does for the entire frame. From Figure 6 , it is clear that the full search algorithm outperformed our previous algorithm in PSNR even though the computational cost has been reduced significantly [1]. However, Figure 7 and 8 show that the algorithm proposed in this paper gives a 1.5dB PSNR gain over FS-Motion and better quality over the previously proposed FS-JM\&D without adding any complexity to the algorithm. Figure 9-10 show similar performance improvements for the whole sequence as well.

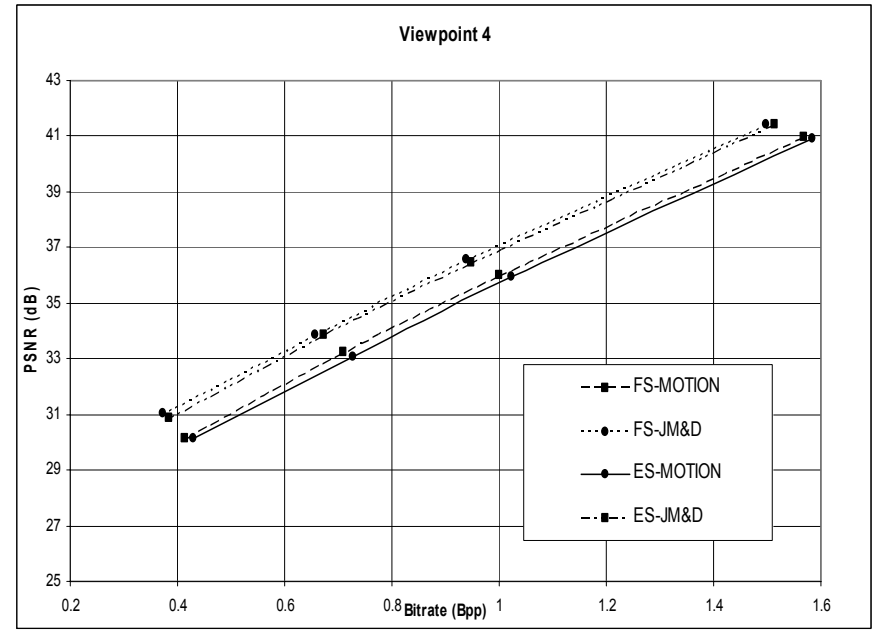

Figure 6 Quality comparisons for viewpoint 4 with ES and full search

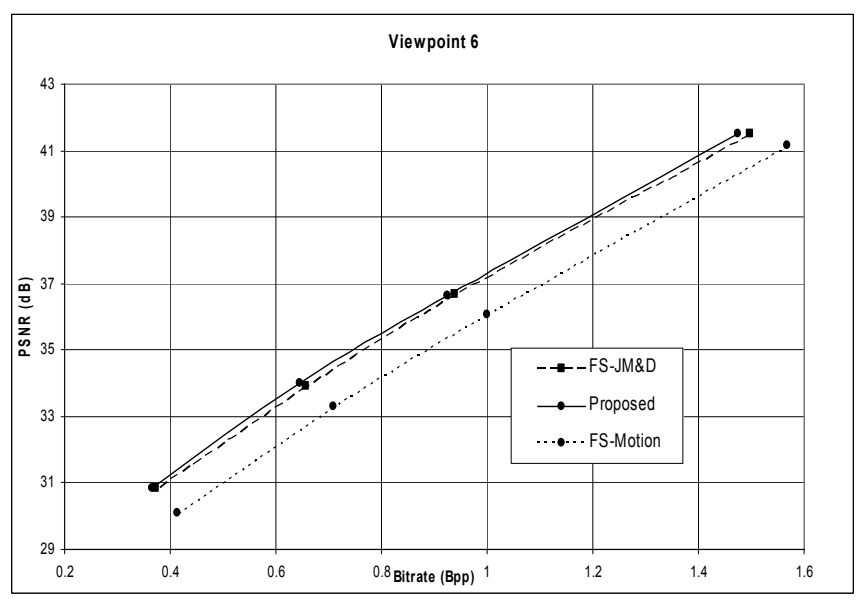

Figure 7 Quality comparison for viewpoint 6

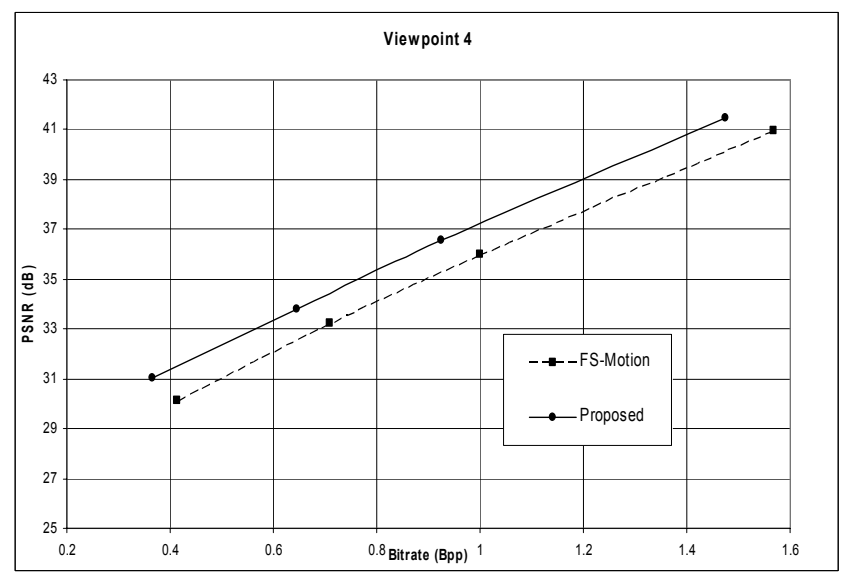

Figure 8 Performance comparison of the proposed algorithm for Viewpoint 4 


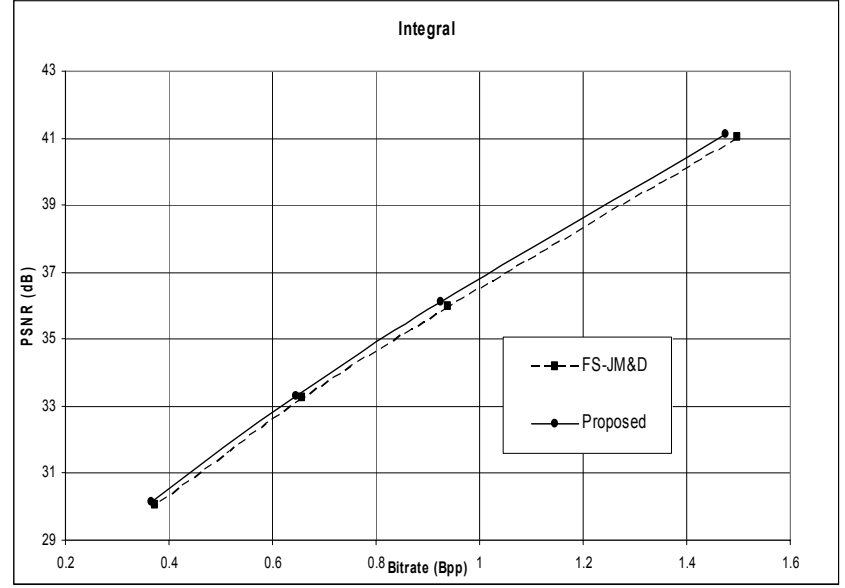

Figure 9 Quality comparison of the average video sequence

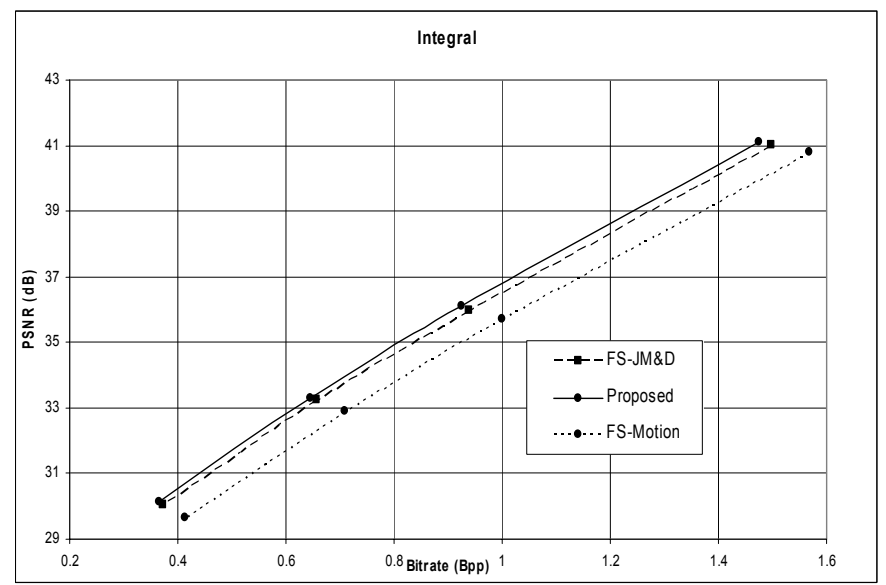

Figure 10 Quality comparison of the average video sequence

\begin{tabular}{|r|c|c|c|}
\hline \multirow{2}{*}{} & \multicolumn{2}{|c|}{ Number of search points } & \multirow{2}{*}{$\begin{array}{c}\text { Complexity } \\
\text { gain }\end{array}$} \\
\cline { 2 - 3 } & Full search & Proposed & \\
\hline Motion & 1024 & 138 & $87.5 \%$ \\
\hline Disparity & 3072 & 302 & $90.1 \%$ \\
\hline
\end{tabular}

Table 1 Complexity comparison of the proposed algorithm

Looking at Figures 6-10 it can be seen that the proposed method gives better coding performance over FS-JM\&D and ES-JM\&D. Furthermore, Table 1 shows that it can also reduce the coding complexity by over $90 \%$ while increasing the video quality.

\section{CONCLUSION}

In this paper, we propose a self-adaptable ES algorithm for joint motion and disparity estimation in $3 \mathrm{D}$ integral video to minimize the computational complexity. The video quality is further improved by half pixel refinement. Experimental results show that the proposed algorithm can achieve similar PSNR with $90 \%$ reduced complexity compared to the full search algorithm. It also gives better quality gain with better coding performance over the previous method [1]. In future, we will be considering early termination criteria for the ES to further improve the performance without significantly reducing the quality.

\section{REFERENCES}

[1] S. Adedoyin, W.A.C. Fernando, A. Aggoun, "A joint motion \& disparity motion estimation technique for $3 \mathrm{~d}$ integral video compression using evolutionary strategy" IEEE Consumer Electronics, Volume: 53, Issue: 2, page(s): 732-739, 2007.

[2] T. Okoshi, T., 'Three-Dimensional imaging techniques', Academic Press, Inc., London, UK. 1976.

[3] L. Onural, et. al., 'An assessment of 3dtv technologies' Proceedings of NAB Broadcast Engineering Conference, pp. 456-467, 2006.

[4] N.A. Dodgson, 'Autostereoscopic 3d displays' IEEE Computer vol. 38(8), pp. $31-36,2005$

[5] M. T.M. Lambooij, W. A. IJsselsteijn, I. Heynderickx: "Visual discomfort in stereoscopic displays: a review" Proc. of SPIE-IS\&T Electronic Imaging, SPIE Vol. 6490, 2007.

[6] N. Davies, M. McCormick and Li. Yang: "Three-dimensional imaging systems: a new development”. Applied Optics. Vol 27, 4520, 1988.

[7] M. McCormick, N. Davies, A. Aggoun, M. Brewin: "Resolution requirements for autostereoscopic full parallax 3d-tv". International Broadcasting Conference, Amsterdam, Sept. 94. IEE Conference Publication No.397, 1994.

[8] S. Manolache, A. Aggoun, M. McCormick, N. Davies, S.Y. Kung, "Analytical model of a three-dimensional integral image recording system that uses circular and hexagonal based spherical surface microlenses", Journal of the Optical Society of America. pt A, 18,No.7, pp 1814-1821, Aug. 2001.

[9] A. Aggoun: 'Pre-processing of integral images for 3d displays' IEEE Journal of Display Technology, Vol. 2. NO. 4, pp. 393-400, 2006.

[10] J. Arai et. al.: "Gradient-index lens-array method based on real time integral photography for three-dimensional images" Applied Optics, No. 11, pp. 2034-2045, 1998.

[11] F. Okano et. al.: "Real time pickup method for a three-dimensional image based on integral photography" Applied Optics, 36, No. 7, pp. 1598-1603, 1997.

[12] J.S. Jang and B. Javidi, "Formation of orthoscopic three dimensional real images in direct pickup one step integral imaging", Optical Engineering, Vol. 42(7), pp. 1869-1870, 2003.

[13] M. Martínez-Corral, B. Javidi, R. Martínez-Cuenca and G. Saavedra, "Formation of real, orthoscopic integral images by smart pixel mapping", Optics Express 13, pp. 9175-9180, 2005.

[14] B. Javidi, R. Martínez-Cuenca, G. Saavedra, and M. Martínez-Corral "Orthoscopic, long-focal-depth integral imaging by hybrid method" Proc. of SPIE Vol. 6392, 639203, 2006.

[15] G. Lippmann, 'Eppreuves reversibles donnat durelief', J. Phys. Paris $821,1908$.

[16] R. Martinez-Cuenca, G. Saavedra, M. Martinez-Corral, B. Javidi, 'Extended depth-of-field 3-d display and visualization by combination of amplitude-modulated microlenses and deconvolution tools' IEEE/OSA Journal of display technology, Vol. 1 (2), pp. 321- 327, 2005.

[17] F. Okano, H. Hoshino, J. Arai and I. Yuyama, 'Real-time pickup method for a three-dimensional image based on integral photography', Apply Optical, Vol. 36, pp.1598-1604, 1997.

[18] M. Martínez-Corral, B. Javidi, R. Martínez-Cuenca and G. Saavedra, 'Formation of real, orthoscopic integral images by smart pixel mapping', Optics Express 13, pp. 9175-9180, 2005.

[19] A Aggoun: 'A 3D DCT compression algorithm for omnidirectional integral images' ICASSP 2006.

[20] R. Zaharia, A. Aggoun and M. McCormick, 'Adaptive 3d-dct compression algorithm for continuous parallax 3d integral imaging' Journal of Signal Processing: Image Communications, Vol. 17(3), pp. 231-242 2002.

[21] R. Zaharia, A. Aggoun and M. McCormick: 'Compression of full parallax colour integral $3 \mathrm{~d}$ tv image data based on sub-sampling of chrominance components" Proceedings of Data Compression Conference, DCC 2001, Snowbird, Utah, USA. IEEE Computer Society, ISBN 0-7695-1031-0, pp. 527,2001. 
[22] Forman, M.C. and Aggoun, A., 'Quantisation strategies for $3 \mathrm{~d} d c t$ based compression of full parallax $3 \mathrm{~d}$ images', IPA97, Conf. Pub. No.443, IEE, 1997.

[23] W.U. ChungHong, "Depth Measurement in integral Images" PhD Thesis, pp. 40-60, 2003.

[24] Charles Darwin, "The origin of species: by means of natural selection or the preservation of favoured races in the struggle for life (bantam classic)," Bantam Classics, Reprint. 1999.

[25] J.M. Fitzpatrick, J.J. Grefenstette, and D. Van-Gucht, "Image registration by genetic search," Proceedings of Southeastcon 84, Louisville, KY, 460-464, Apr 1984.

[26] Ingo Rechenberg, Evolutionsstrategie '94. Stuttgart: FrommannHolzboog 1994.

[27] Garrison W. Greenwood, Qiji J. Zhu "Convergence in evolutionary programs with self-adaptation" evolutionary computation, v.9 n.2, p.147-157, June 2001.

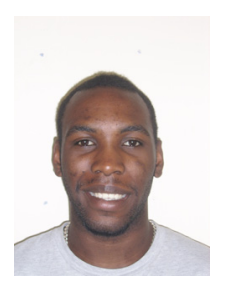

S. Adedoyin is a research student at University of Surrey, UK. He holds a BEng (Hons) in Microelectronics Engineering that was obtained from Brunel University, UK. His current research interest lies in 3D integral imaging, 3D video coding and H.264.

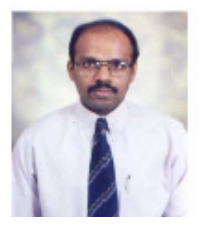

W.A.C. Fernando received the B.Sc. Engineering degree (First class) in Electronic and Telecommunications Engineering from the University of Moratuwa, Sri Lanka in 1995 and the MEng degree (Distinction) in Telecommunications from Asian Institute of Technology (AIT), Bangkok, Thailand in 1997. He completed his $\mathrm{PhD}$ at the Department of Electrical and Electronic Engineering, University of Bristol, UK in February 2001. Currently, he is a senior lecture in signal processing at the University of Surrey, UK. Prior to that, he was a senior lecturer in Brunel University, UK and an assistant professor in AIT. His current research interests include Distribute Video Coding (DVC), 3D video coding, intelligent video encoding for wireless communications, OFDM and CDMA for wireless channels, channel coding and modulation schemes for wireless channels. He has published more than 150 international papers on these areas. He is a senior member of IEEE and a fellow of the HEA, UK. He is also a member of the EPSRC College.

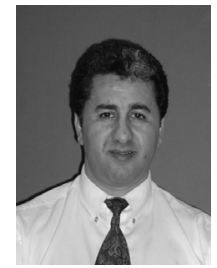

A. Aggoun (M' 99) received the "Ingenieur d'Etat" degree in electronic engineering from Ecole Nationale Polytechnique of Algiers (ENPA) Algeria, in 1986 and the $\mathrm{PhD}$ degree in compressed video signal processing from Nottingham University, UK, in 1991. From 19911993 he was with the Nottingham University as a research fellow in digital video signal processing. From 1993-2005, he was with De Montfort University, UK, as a Principle lecturer in Electronic Engineering. In 2005, he joined the school of Design and Engineering at Brunel University (UK) as a Reader in 3D Imaging Technologies.

His current research Interests include computer generation and live capture of 3D integral images, 3DTV, 3D visualisation, depth measurement and volumetric data reconstruction from 3D integral images, 3D video coding, computer vision, and real-time digital image processing architectures.

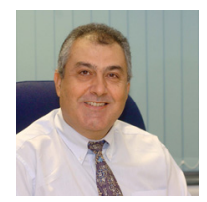

A.M. Kondoz received the B.Sc. (Hons.) degree in engineering, the M.Sc. degree in telematics, and the Ph.D. in communication in 1983, 1984, and 1986, respectively. He became a Lecturer in 1988, a Reader in 1995, and then in 1996, a Professor in Multimedia Communication Systems and deputy director of Centre for Communication Systems Research (CCSR), University of Surrey, Guildford, U.K. He has over 250 publications, including a book on low bit-rate speech coding and several book chapters. He has graduated more than $40 \mathrm{Ph}$.D. students in the areas of speech/image and signal processing and wireless multimedia communications, and has been a consultant for major wireless media terminal developers and manufacturers. Prof Kondoz has been awarded several prizes, the most significant of which are The Royal Television Societies' Communications Innovation Award and The IEE Benefactors Premium Award. He has been on the Refereeing College for EPSRC and on the Canadian Research Councils. He is a member of the IEEE and the IET. 